\title{
Drain Temperature Dependence on Ambient Temperature for a Cryogenic Low Noise C-Band Amplifier.
}

\author{
S. Muñoz, J. D. Gallego', J. L. Sebastián and J.M. Miranda. \\ Dpt. Fisica Aplicada III \\ Facultad de Ciencias Físicas. U. C. M. \\ 28040 Madrid ( Spain). \\ ' Centro Astronómico de Yebes. \\ Aptdo. 148. E19080 Guadalajara ( Spain).
}

\begin{abstract}
A comparison between predicted and measured noise temperatures for cryogenic HEMT amplifiers is presented by using the Pospieszalski's noise model. A good agreement between predicted and measured amplifier's noise performance is obtained both at room and cryogenic temperatures. However, the predicted values overestimate noise temperature in the center part of the measured temperature range $(50 \mathrm{~K}-230 \mathrm{~K})$. A parabolic dependence for the drain temperature with ambient temperature is proposed to obtain a better fitting to the experimental results.
\end{abstract}

\section{INTRODUCTION}

In order to reduce the number of measurements needed to obtain noise figure predictions the analysis of the FETs' (MODFETs) noise performance has became a subject of primary interest. It has already been shown (1) that a complete noise analysis can be performed using a simple noise measurement and an equivalent circuit model. More recently $(2,3)$, other models have been described that further simplify the measurement requirements while improving the noise figure predictions. These models predict the four noise parameters at any frequency provided that two frequency independent constants and the transistor's equivalent circuit are known. These two constants are the equivalent temperatures of the intrinsic gate resistance and drain conductance, $T_{g}$ and $T_{d}$ respectively.

Verifications of these models have already been carried out with noise and S-parameters data taken for different active devices at a single operating bias point and as a function of operating bias (4). The validity of a simplified noisy model has recently been checked for packaged pseudomorphic devices by comparison between the measured noise parameters with those obtained from a computer analysis of the simplified noisy model of the device (5).

The values of $T_{d}$ and $T_{g}$ are known for typical HEMT devices (6) at room and cryogenic temperatures. Our previous simulations assumed that $T_{g}$ was very close to the transistor's physical temperature and that $T_{d}$ could be obtained from linear interpolation of the values known at room and cryogenic temperatures (297K and $14 \mathrm{~K}$ respectively) (7).

This work shows a comparison between predicted and measured noise temperature for cryogenic HEMT amplifiers designed over the frequency range $3.2 \mathrm{GHz}-4.7 \mathrm{GHz}$. The noise temperature is simulated by using the model proposed by Pospieszalski (3). The experimental results suggest a non linear dependence of $T_{d}$ on ambient temperature since a linear interpolation overestimates $T_{n}$ at ambient temperatures in the range $50 \mathrm{~K}-230 \mathrm{~K}$. 
Four cryogenic C-band amplifiers have been designed and built with transistors from different manufactures and batches. Each amplifier consists of three stages as shown in Figure 1.

The source inductance and loading resistor values were selected for each stage so that both unconditional stability and low power dissipation across the full band were achieved. Therefore, no oscillation was produced for any combination of passive input and output impedances at any operating temperature. The input matching circuit was optimized for minimum cryogenic noise and the output matching circuit for low reflection. The two interstage coupling networks provide flat gain of approximately $34 \mathrm{~dB}$ for the amplifier.

The noise temperature was measured with a fully automated system in the range $15 \mathrm{~K}-275 \mathrm{~K}$. Cryogenic measurements were taken with the "cold attenuator" method by using a calibrated noise diode at room temperature, a $15 \mathrm{~dB}$ attenuator and a DC-block cooled at cryogenic temperatures. Room temperature data were obtained with the noise diode. The accuracy of measured noise temperature is estimated in $\pm 9 \mathrm{~K}$ for the room temperature and $\pm 1 \mathrm{~K}$ for the lowest cryogenic temperature, in the worst case (8).

All the amplifiers were biased for minimum noise operation at room temperature $\left(\mathrm{V}_{\mathrm{ds}}=2 \mathrm{~V} ; \mathrm{I}_{\mathrm{ds}}=\right.$ $10 \mathrm{~mA}$ ). When the amplifiers were cooled below $100 \mathrm{~K}$, the bias was changed to a new value of $\mathrm{V}_{\mathrm{ds}}=$ $1.5 \mathrm{~V} ; \mathrm{I}_{\mathrm{ds}}=5 \mathrm{~mA}$ which corresponds to the optimum noise for $\mathrm{T}_{\mathrm{amb}}=15 \mathrm{~K}$.

Figure 2 shows the measured mean noise temperature for the four amplifiers as a function of ambient temperature in the range $15 \mathrm{~K}-270 \mathrm{~K}$.

As is observed, amplifiers 1 and 2 show the best performance with a mean noise temperature of approximately $10 \mathrm{~K}$. $\mathrm{T}_{\text {amb }}=60 \mathrm{~K}$ with a ripple of $\pm 0.5 \mathrm{~K}$ in the band.

Amplifiers 3 and 4 had an anomalous performance showing a bump around 125K that may be due to trap effects at cryogenic temperatures.

\section{MODELING NOISE TEMPERATURE.}

The amplifiers were simulated with MMICAD (9) by using the Pospieszalski's noise model and a HEMT equivalent circuit obtained from DC and $S$ parameters measurements of the active device at room temperature. Capacitors were modeled as RCL circuits and resistors as RL circuits. In order to obtain unconditional stability and an acceptable input reflection across the full band, an inductive feedback was used at the source and the drain was resistively loaded. The noise added by the drain loading resistors to the total noise of the amplifiers is $0.4 \mathrm{~K}$ for $T_{\text {amb }}=15 \mathrm{~K}$ and $10 \mathrm{~K}$ for $T_{\text {amb }}=300 \mathrm{~K}$. This noise represents about $10 \%$ of the total noise at cryogenic temperature and $20 \%$ at room temperature.

In the Pospieszalski's noise model, the HEMT's noise properties are only determined by the values of the equivalent gate and drain temperatures $T_{g}$ and $T_{d}$. The equivalent gate temperature is considered to be equal to the ambient temperature as typically shown by HEMT devices (3). In order to model the noise temperature of the amplifiers at any ambient temperature, $T_{d}$ is obtained as a first approximation by using a linear interpolation from the temperature values originally proposed by Pospieszalski $(4,6)$. However, the linear interpolation does not predict the experimental data accurately and therefore another approach has been considered. The experimentally observed deviation from linearity and the overestimation of the noise at high temperatures suggested us that a non linear dependence of $T_{d}$ with 
$\mathrm{T}_{\text {amb }}$ may improve the predictions. The parabolic dependence shown in Fig. 3 may be easily included in MMICAD.

Figure 4 shows a comparison between predicted (with linear and non linear dependence) and measured noise temperature for all amplifiers as a function of ambient temperature in the range $15 \mathrm{~K}-270 \mathrm{~K}$.

As is observed, the non linear drain temperature dependence on ambient temperature predicts the best fitting to measured mean noise temperature in the center part of temperature range. A better value for amplifiers noise performance at room temperature is also provided by the non linear dependence.

\section{CONCLUSIONS}

As observed in Figure 4, a parabolic dependence of $T_{d}$ predicts a good fitting of the noise temperature in the ambient temperature range $15 \mathrm{~K}$ to $300 \mathrm{~K}$. Pospieszalski's values were correct for $T_{a m b}=15 \mathrm{~K}$ but they overestimate the noise at room temperature. This may be due to the improvement of the devices presently available. The results shown in Figure 4 suggest that the new proposed $T_{d}$ function leads to a more accurate prediction of the noise temperature of cryogenic amplifiers in a broad ambient temperature range, modeling the nonlinear dependence of $T_{n}$ with $T_{\text {amb }}$.

\section{REFERENCES}

(1) A. F. Podell. "A Functional GaAs FET Noise Model". May 1981, IEEE Trans on ED, Vol. 28, pp. 511-517.

(2) M. S. Gupta and P. T. Greiling. "Microwave Noise Characterization of GaAs MESFET's: Determination of Extrinsic Noise Parameters". Apr. 1988, IEEE Trans. on MTT, Vol. 36, pp. 745-751.

(3) M. Pospieszalski. "Model of Noise Parameters of MESFETs and MODFETs and their Frequency and Temperature Dependence". Sept. 1989. IEEE Trans. on MMT, Vol. 37, pp. 1340-1350.

(4) M. W. Pospieszalski and A. C. Niedzwiecki. "FET Noise Model and On-Wafer Measurement of Noise Parameters". Proc. of 1991 Int. Microwave Symp.

(5) A. Caddemi, A. di Paola and M. Sanino. "Full Characterization of Microwave Low-Noise Packaged HEMT's: Measurements Versus Modeling". Apr. 1997, IEEE Trans. Instrum. Meas. Vol. 42, $\mathrm{n}^{\circ}$ 2, pp. 490-494.

(6) M. Pospieszalski. "Cryogenically-Cooled HFET Amplifiers and Receivers: State of the Art and Future Trends". 1992, IEEE MTT-S Digest. pp. 1369-1372.

(7) M. Pospieszalski et al. "Very Low Noise and Low Power Operation of Cryogenic AlInAs/GaInAs/InP HFET's". 1994, IEEE MTT-S Digest. pp. 1345-1346.

(8) J. D. Gallego. "Cryogenic C-Band HEMT Amplifier Prototypes For IRAM". Technical Report CAY 1.

(9) MMICAD Ver. 5.20. Monolithic and Microwave Integrated Circuit Analysis and Design, 1991, Optotek Ltd., 62 Steacie Drive, Kanata, Ontario K2K2A9, Canada. 


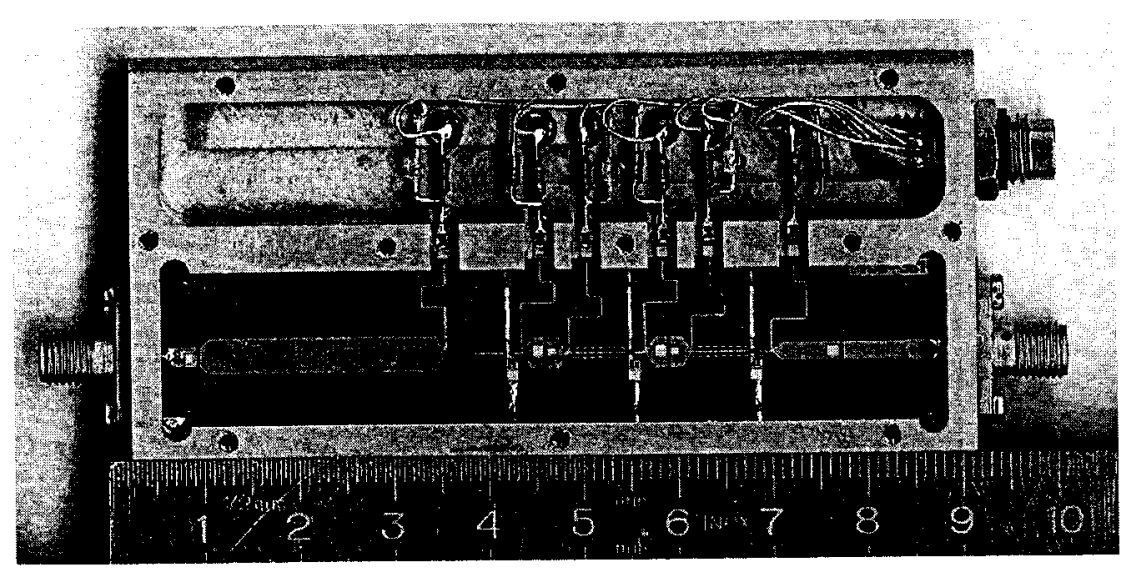

Fig. 1. A three stage cryogenic C-band amplifier.
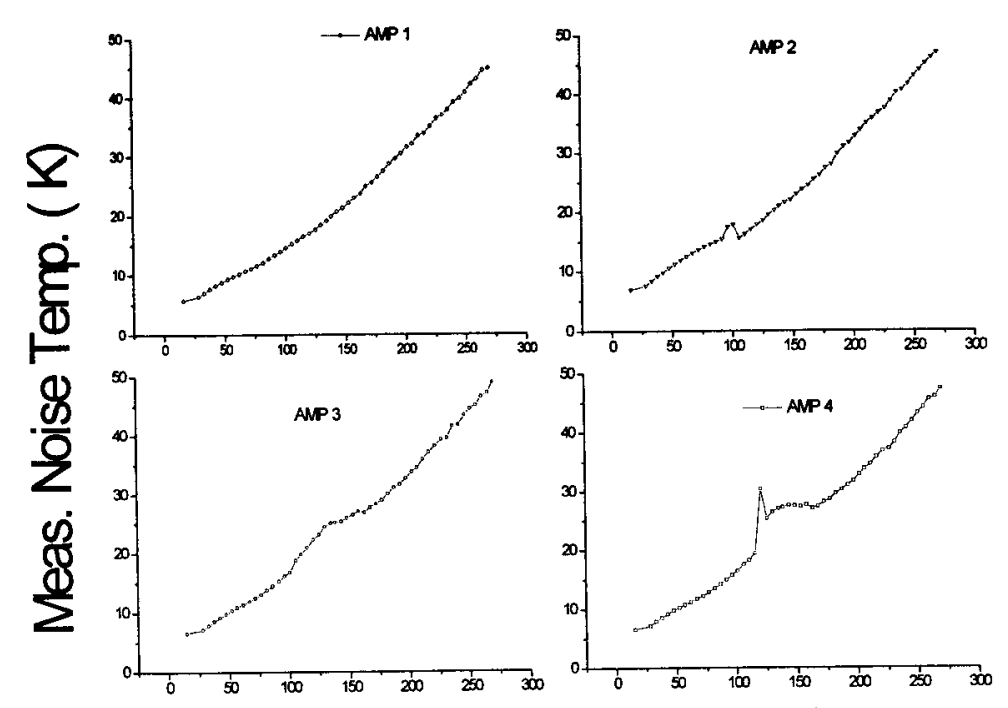

Ambient Temperature $(\mathrm{K})$

Fig 2. Noise temperature of different cryogenic C-band amplifiers. 


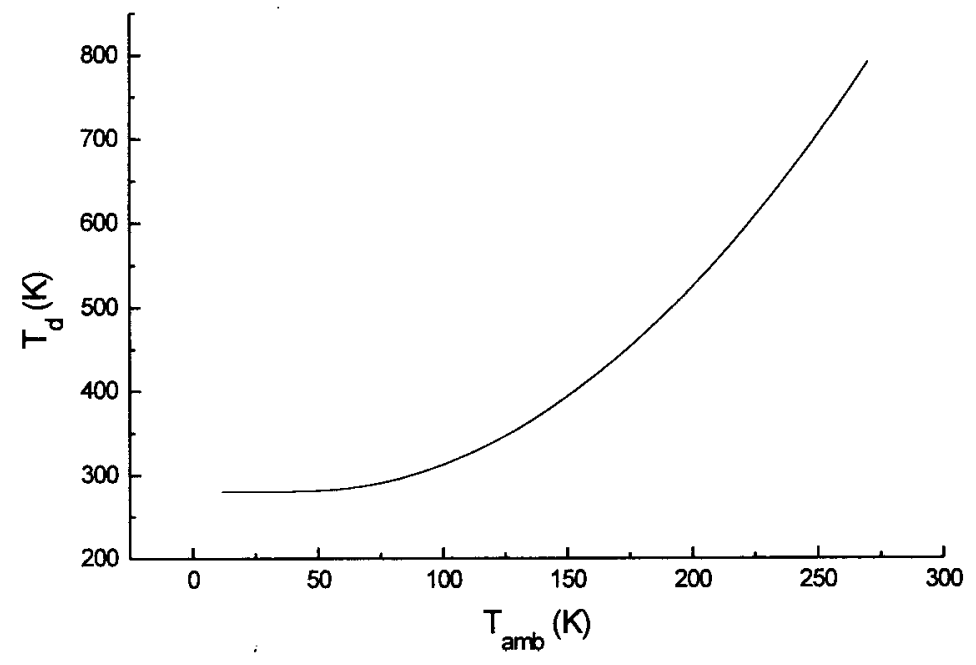

Fig. 3. Drain temperature non linear dependence on ambient temperature.

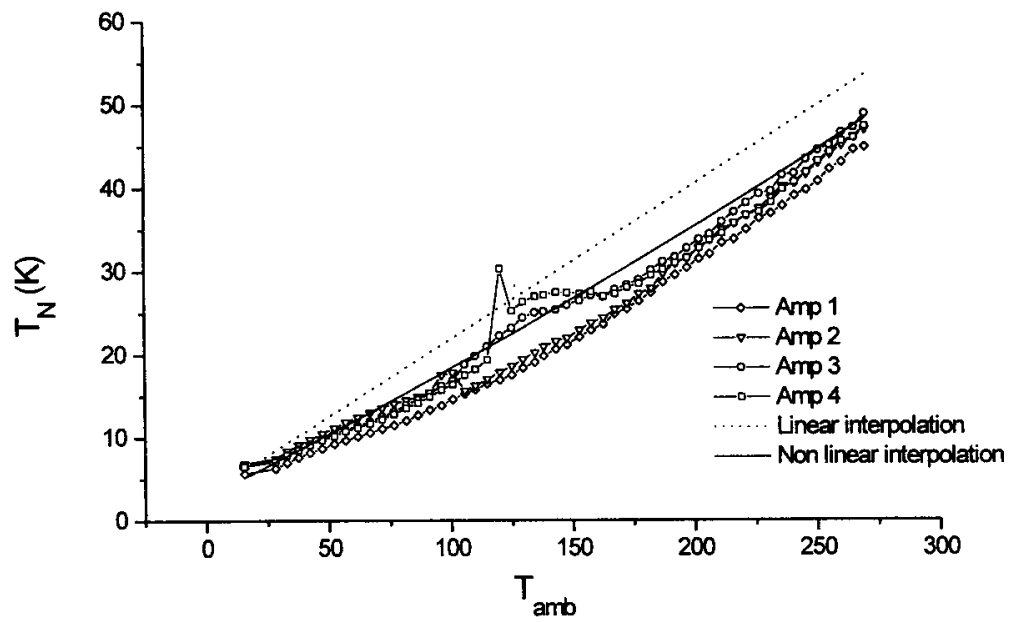

Fig. 4. A comparison between predicted and measured noise temperatures for cryogenic HEMT amplifiers. 